\title{
Sleep Disturbance in Elderly Patients after Cardiac Surgery during their Stay in Intensive Care Unit and Surgical Ward
}

Hiromi Arita ${ }^{1,2^{\star}}$, Yukari Takeno ${ }^{3}$, Etsuko Fujimoto ${ }^{4}$

${ }^{1}$ Associate professor, Department of Faculty of Nursing and Social Welfare Sciences, Fukui Prefectural University, Japan ${ }^{2}$ Graduate School of Medicine, Nagoya University, Japan

${ }^{3}$ Assistant Professor, School of Nursing \& Health, Aichi Prefectural University, Japan

${ }^{4}$ Professor, Department of nursing, Graduate School of Medicine, Nagoya University, Japan

\section{Abstract}

Background: Sleep patterns of patients who admitted the intensive care unit (ICU) after surgery have shown fragment and disturbed sleep during early postoperative days. However, there is less information about sleep status in patients after transferred to a surgical ward from the ICU. The aim of this study was to evaluate the sleep patterns in elderly patients after cardiac surgery including stay in both the ICU and surgical ward.

Methods: Twelve patients aged $\geq 65$ years were examined for 3 days before and 6 days after surgery. We used to examine sleep quality an actigraph and the Oguri-Shirakawa-Azumi sleep inventory (version for patients of middle age and older) for sleep.

Results: Total sleep time was significantly decreased postoperative day 3 (median, $368.5 \mathrm{~min}$ ) and 4 (median, $433.5 \mathrm{~min}$ ) than before surgery (median $526.0 \mathrm{~min})(p<0.05)$. Nocturnal sleep time showed significant decreases from preoperative values until postoperative day 1-4 $(p<0.05)$. Duration of longest sleep was significantly decreased postoperative day 1-3 than before surgery $(p<0.05)$. Sleep scale (sleep length and maintenance of sleep) scores indicated a decline in sleep quality on POD3 compared with preoperative values $(p<0.05)$. The sleep disturbances did not reach preoperative levels until at least POD4.

Conclusions: The sleep disturbances persisted not only throughout patients' ICU stay but also after they had been discharged from the ICU. Therefore, close collaboration between surgical ward and ICU nurses are the key to provide more appropriate and early interventions.

Keywords: Elderly patients; Cardiac surgery; Sleep pattern; Actigraph

\section{Introduction}

Although cardiac surgery has become common, even for the elderly, because of recent advances in medical technology, elderly patients are likely to suffer serious risks and complications associated with this invasive surgical technique. In addition, those hospitalized in the Intensive Care Unit (ICU) after cardiac surgery for routine postoperative care may experience multiple stresses because of various factors such as environmental noise in the ICU, diagnostic tests, the after-effects of general anesthesia, and pain [1-6]. Sleep/wake patterns may be disturbed because of these stress factors [7-14], and sleep is extremely important for postoperative physical recovery not only in the ICU but also in the surgical ward after discharge from the ICU.

There are four primary methods to measure sleep in the postoperative patient, i.e., by polysomnography, actigraphy, nurses' observation, and questionnaire methods. Previous studies revealed the following alteration in sleep architecture by polysomnography: stage 1 and 2 sleep increased and stage 3 and 4 , and rapid eye movement sleep decreased $[7,8,11]$. These studies refer to changes in the sleep for only 24-48 hours in the ICU at a hospital. In contrast, actigraphy is also used for researching sleep in patients who underwent abdominal or cardiac surgeries. Previous studies indicated that nocturnal sleep time and efficiency decreased, total sleep time (TST) either decreased or no change was observed, and frequent nocturnal arousal increased on actigraphy, which was performed within the periods of 2-3 days after cardiac surgery $[12,13,15]$. Therefore, it is difficult to gain knowledge on the sleep of elderly patients during their stay in ICU and surgical ward.

Nurse clinically uses their observations to assess a patient's sleep. However, ICU nurses tend to overestimate the quality of patients' sleep pattern [16]; nursing records often only contain entries on the patient's physical condition, and information about sleep disturbance is minimal [17]. Therefore, surgical ward nurses have no way of knowing patients' sleep problems. For nursing interventions to be effective, detailed analyses of patients' sleep patterns are required based on observations on consecutive days. However, there are a few studies on sleep deprivation after ICU discharge in elderly patients and recent research that investigated postoperative sleep using actigraph.

In this study, we report the sleep patterns in elderly patients from 3 days before and 6 days after cardiac surgery including stay in both the ICU and surgical ward. The results of this study may alter nurses' awareness of their patients' sleep patterns, aiding them in providing preventive care against sleep disorders in both the ICU and surgical ward.

\section{Materials and Methods}

\section{Participants}

Participants were patients who underwent cardiac surgery at the Fukui Cardiovascular Center (199 beds included 9 beds of ICU), which is the only cardiac specialty hospital in Fukui Prefecture. The hospital undertakes more than 400 cardiac surgeries every year, and the average duration of hospital stay of patients undergoing cardiac surgery is approximately 16 days. Patient inclusion criteria were as follows: age of

*Corresponding author: Hiromi Arita, Associate professor, Department of Faculty of Nursing and Social Welfare Sciences, Fukui Prefectural University, 4-1-1 Kenjojima, Matsuoka, Eiheiji-Town, Fukui 910-1195, Japan, Tel: +81-776-61-6000 (Ex.4413); Fax: +81-776-61-6016; E-mail: arita@fpu.ac.jp

Received August 07, 2013; Accepted November 07, 2013; Published November 11,2013

Citation: Arita H, Takeno Y, Fujimoto E (2013) Sleep Disturbance in Elderly Patients after Cardiac Surgery during their Stay in Intensive Care Unit and Surgical Ward. J Nurs Care 3: 137. doi:10.4172/2167-1168.1000137

Copyright: ( $) 2013$ Ekström A, et al. This is an open-access article distributed under the terms of the Creative Commons Attribution License, which permits unrestricted use, distribution, and reproduction in any medium, provided the original author and source are credited. 
$>65$ years; hospital admission for nonemergency surgery; no psychosis or dementia disorders; capable of comprehending and replying to questions; no previous history of sleep disorders, as indicated by scoring on the Pittsburgh Sleep Quality Index (PSQI, Japanese version); and no sleep-enhancing medications. This study was conducted from October 2011 to March 2012 and was approved by the Research Ethics Committees of Nagoya University and Fukui Cardiovascular Center. All patients provided written informed consent before participating in the study.

\section{Instruments}

The sleep instrument used in this study was an actigraph (Ambulatory Monitoring, Inc., NY), and we utilized the OguriShirakawa-Azumi Sleep Inventory (OSA-MA; version for middle-aged and older patients).

Objective sleep indicators: A wrist actigraph was used as an objective measure of sleep in this study. The actigraph, which is device that senses physical motion and is worn on the wrist, allows measurement of patients over several days and nights with less intrusiveness than polysomnography. The collected data were downloaded to a computer for analysis and estimated using Cole's algorithm: automatic scoring algorithms developed by Cole, et al. [18] have indicated high agreement between actigraphy and polysomnography for sleep and wakefulness. Therefore, actigraphy is now widely used in research studies including those on ICU patients. Although an actigraph is easy to use and helpful in practical terms, it can be influenced by restricted body movement. We used actigraph because our participants had no limitation of body movement such as body fixation or mechanical ventilation. The following sleep variables were evaluated:

TST: sleep time during a 24-hour period.

Nocturnal sleep time: total sleep from 21:00 to 05:59

Daytime sleep time: total sleep from 6:00 to 20:59

Duration of longest sleep: duration of longest sleep from 21:00 to 05:59

Awakening during sleep: awake time from 21:00 to 05:59

Sleep efficiency is often used as an indicator for measuring the quality of sleep. However, because most participants did not sleep well at night, it was determined that this parameter would be inappropriate in the present study. Actigraph data were partitioned into daytime (06:00 to 20:59) and nighttime (21:00 to $05: 59)$; these intervals were arranged to coincide with daily hospital routines.
Subjective sleep indicators: The OSA-MA questionnaire was developed in Japan and has been used to evaluate subjective sleep quality. It includes 5 factors combining of a total of 16 items: factor I, "sleepiness on rising" ( 4 items); factor II, "initiation and maintenance" (5 items); factor III, "frequent dreaming" ( 2 items); factor IV, "feeling refreshed" (3 items); and factor V, "sleep duration" ( 2 items). Individual items are scored on a 4-point Likert-type scale, with higher scores indicating better sleep quality. Its validity and reliability have been tested in an elderly Japanese population (Cronbach's alpha 0.81) [19]. The OSA-MA questionnaire has been used in Japan to study sleep in patients in the postoperative setting [20].

\section{Procedure}

Patients usually receive care in the ICU for approximately 3 days after cardiac surgery before being transferred to a surgical ward in our institution. Therefore, this study was conducted from 3 days before surgery to 6 days after transfer to a surgical ward from the ICU (Figure 1). The actigraph was used on the patient's nondominant wrist. It was removed on the morning of the operative day and reapplied after ICU admission. Actigraph data from the day before the surgery and postoperative day (POD) 0 (i.e., the day of surgery) were excluded because of the potential effects of sleep medication or anesthesia.

The OSA-MA questionnaire was self-reported in the morning regarding sleep the previous night on the 3 days before surgery. However, data of the night before surgery were excluded in order to administer sleep medication as per preoperative hospital routine. The investigator interviewed patients on the morning of POD 3 and 5 because of ethical considerations and the potential effects of cognition anesthesia.

Illumination in the hospital room was measured by a light sensor (Ambulatory Monitoring, Inc. USA). In both the ICU and surgical wards, room lights were turned off at 21:00 and illumination was maintained at 0-20 lux at pillow height. Room lights were turned on at 06:00. Illumination was maintained at 800-1000 lux in the ICU and at 1500-2000 lux in the surgical wards (private room).

\section{Data analysis}

Actigraph data were downloaded to a personal computer and analyzed by Action4 software (Ambulatory Monitoring, Inc. NY). Preoperative (preoperative day 3 and 2) actigraphy and OSA-MA variables were averaged as baseline, then actigraphy variables were statistically compared between baseline and POD 1, 2, 3, and 4. OSAMA variables were compared between baseline and POD 3 and 5. The significance of differences was statistically analyzed with repeated

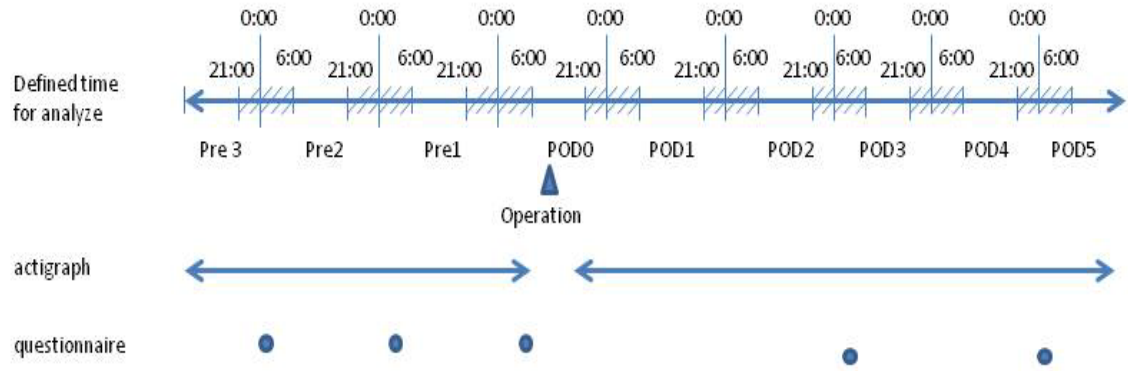

Figure 1: Measurement procedure

Operative day $(\boldsymbol{\Delta})$. Evaluations were made for 9 consecutive days, including 3 days before surgery and 6 days after surgery. We defined $06: 00-21: 00 \mathrm{~h}$ as daytime and 21:00-06:00 $\mathrm{h}$ as nighttime. The operative day refer to POD 0 , the following days refer to POD 1-5. 
Citation: Arita H, Takeno Y, Fujimoto E (2013) Sleep Disturbance in Elderly Patients after Cardiac Surgery during their Stay in Intensive Care Unit and Surgical Ward. J Nurs Care 3: 137. doi:10.4172/2167-1168.1000137

Page 3 of 6

measures by ANOVA (Friedman's tests). When a significant difference was observed, the Bonferroni method was used as a post hoc test. Data are presented as mean \pm standard divisions (SD). Statistical analyses were performed using SPSS 19.0J for Windows (SPSS Japan, Tokyo, Japan). A $p$ value of $<.05$ was considered statistically significant.

\section{Results}

\section{Demographic and clinical data}

A convenience sample of 12 patients participated in this study (Table 1 ). The mean \pm standard divisions (SD) in age were $74.8 \pm 5.3$ years (range, 68 83 years). Coronary artery bypass graft (CABG), including both CABG and valve repair was performed in the majority of participants $(n=9)$; others had undergone valve replacements or repair. The mean duration of anesthesia and surgery was $329.2 \pm 86.1$ and $258.3 \pm 83.3 \mathrm{~min}$, respectively. The mean length of ICU stay was $2.5 \pm 1.1$ days. All participants were transferred from the ICU to a surgical ward on POD4. Although participants arrived in the ICU mechanically ventilated, they were extubated within $4-12 \mathrm{~h}$ after surgery, and their chest tubes were removed 2-3 days after surgery. During the first 48 hours after surgery, low doses of fentanyl were administered by intravenous infusion for pain management. No complication directly related to the surgery was evident, and no participant developed delirium.

\section{Actigraph records}

The sleep-wakefulness pattern of 1 participant is shown in Figure
2. Three days before surgery, this participant's nocturnal sleep started after lights were turned off at 21:00 and continued sleeping until the following morning. A large amount of activity was recorded during the daytime. Regular sleep cycles were recorded during the 3 preoperative days of observation. However, after surgery, regular sleep cycles were disrupted. From POD1-3 the participant was unable to sleep well, during the night of POD4, sleep was fragmented. Actigraph patterns for other 5 participants were similar.

\section{TST}

The mean \pm SD of baseline was $506.7 \pm 102.4 \mathrm{~min}$. TST increased to $655.2 \pm 355.8 \mathrm{~min}$ on POD1 (Figure 3), and thereafter, dropped to $442.9 \pm 234.8 \mathrm{~min}$ on POD2. A statistically significant difference was noted between baseline and POD3 values $(339.0 \pm 133.8 \mathrm{~min})(p<.05)$. Similarly, a significant difference was detected between baseline and POD4 values $(406.8 \pm 104.0 \mathrm{~min})(p<.05)$. An analysis of individual participant data indicated that 10 of the 12 participants had not reached their baseline (i.e., preoperative) by POD 4 . In one of the two remaining participants, TST gradually decreased until POD3 and reached his baseline on POD4; in the remaining participant, whose baseline TST was less than that of others, TST stabilized until POD4.

Nocturnal and daytime sleep time: TST was divided into nocturnal and daytime periods for comparison (Figure 3). The mean of nocturnal sleep time was $445.4 \pm 85.5 \mathrm{~min}$ on baseline. Postoperative nocturnal

\begin{tabular}{|c|c|c|c|c|c|c|c|}
\hline ID & Age & Sex & Type of surgery & Anesthesia (min) & Operation (min) & ICU stay (day) & Medication (fentanyl) \\
\hline 1 & 83 & $\mathrm{~F}$ & CABG & 240 & 165 & 1 & 1day \\
\hline 2 & 68 & M & CABG & 285 & 215 & 2 & 2days \\
\hline 3 & 70 & M & CABG + valvuloplasty & 510 & 435 & 3 & 2days \\
\hline 4 & 73 & M & CABG + valvuloplasty & 320 & 255 & 4 & 2days \\
\hline 5 & 71 & M & CABG + valvuloplasty & 385 & 300 & 3 & 2days \\
\hline 6 & 68 & $\mathrm{M}$ & Pacth closure & 245 & 180 & 1 & 1day \\
\hline 7 & 81 & $\mathrm{~F}$ & CABG & 255 & 180 & 1 & 2days \\
\hline 8 & 71 & M & CABG & 405 & 330 & 4 & 2days \\
\hline 9 & 80 & $\mathrm{~F}$ & CABG & 290 & 225 & 3 & 2days \\
\hline 10 & 76 & $\mathrm{~F}$ & Valve replacement & 245 & 185 & 2 & 1day \\
\hline 11 & 79 & M & valvuloplasty & 350 & 280 & 3 & 2days \\
\hline 12 & 77 & $\mathrm{M}$ & CABG + valvuloplasty & 420 & 350 & 3 & 2days \\
\hline
\end{tabular}

Medication was cancelled in a day, if participants have nausea.

Table 1: Characteristics ofhe participants

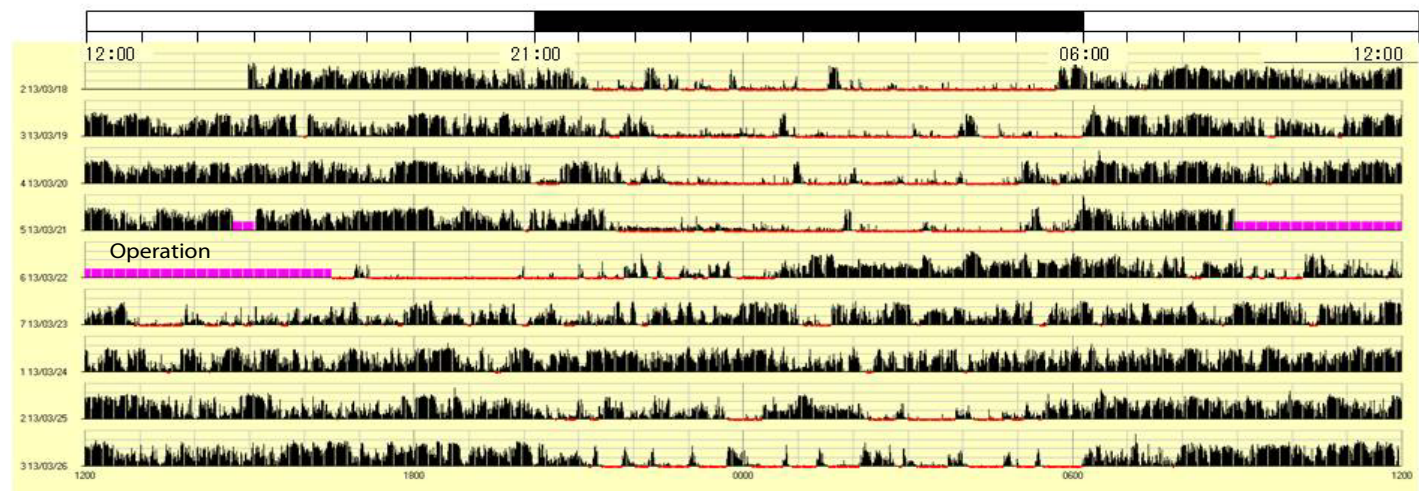

Figure 2: Sample of a subject's actigraph records.

The vertical axes list the observation days and show the activity counts. The horizontal axes express the time of day from 12 a.m to 12 p.m. Top three lines show preoperative sleep/activity. Bottom four lines show postoperative sleep/activity. Regular sleep cycles were noted for 3 days before surgery. However the participant was not able to sleep well from the first postoperative day to the third postoperative day. 
sleep time showed significant decreases from the baseline on POD1 (365.8 $\pm 146.7 \mathrm{~min})$, POD2 (206.6 $\pm 140.3 \mathrm{~min})$, POD3 (225.8 \pm 108.6 $\mathrm{min})$, and $4(283.5 \pm 104.5 \mathrm{~min})(p<.05)$. The lowest nocturnal sleep time was recorded on POD2, which was almost $50 \%$ below the mean baseline value. An analysis of individual participant data indicated a decrease in nocturnal sleep time in all participants, with 2 participants showing a $25 \%$ reduction below baseline throughout the postoperative period. Even by POD4, nocturnal sleep in 11 participants did not reach to the baseline.

Baseline of daytime sleep time was $61.3 \pm 44.0 \mathrm{~min}$. Although postoperative daytime sleep time was significantly higher than the baseline at POD1 (373.8 $\pm 258.7 \mathrm{~min})$ and $2(236.3 \pm 148.5 \mathrm{~min})$ (Figure 2 ), it gradually returned to baseline thereafter. No statistically significant difference from baseline was observed in daytime sleep time after POD3. An analysis of individual participant data indicated that daytime sleep time in 4 participants returned to baseline values, whereas in the other 8 participants, it remained above baseline until POD4.

\section{Duration of longest sleep and awakening during sleep:}

Baseline of duration of longest sleep was $146.5 \pm 64.5$ min (Figure 4). A statistically significant difference from the baseline was observed on POD1 (66.8 $\pm 48.8 \mathrm{~min})$, POD2 (50.6 $\pm 43.6 \mathrm{~min})$, and POD3 (54.3 \pm $22.6 \mathrm{~min})(p<.05)$. An analysis of individual participant data revealed a decrease in the duration of the longest sleep in 10 of the 12 participants until POD4 $(67.6 \pm 33.3 \mathrm{~min})$. The mean number of awakenings during sleep baseline and after surgery was 5 and 9-10, respectively. A statistically significant difference was found between baseline and the values on POD1, 2, 3, and $4(p<.05)$. An analysis of individual participant data demonstrated an increase in nocturnal wakefulness until POD4 in 11 of the 12 participants.

\section{Subjective sleep quality}

OSA-MA scores were presented as mean $\pm \mathrm{SD}$. Baseline scores for factor V, "sleep length," was $25.6 \pm 5.2$. However, this score dropped to $11.0 \pm 7.9$ on POD3 and improved slightly to $18.7 \pm 5.1$ on POD5 (Table 2). These scores show significant differences between the baseline score and score on POD 3 or POD5 $(p<.05)$. Baseline score for factor II, "initiation and maintenance of sleep," was $18.8 \pm 5.5$; this score decreased to $12.6 \pm 5.6$ on POD3 and recovered to $18.8 \pm 8.5$ on POD5. The score for factor II on POD 3 was significantly lower than the baseline scores $(p<.05)$. There were no significant differences between baseline and postoperative scores for other factors. In a previous study on 296 healthy people (75 years $\geq$ age $\geq 60$ years), the mean score for factor V, "sleep length," was $23.9 \pm 6.7$, and the mean score for factor II, "initiation and maintenance of sleep," was $20.9 \pm 6.8$ [16].

\section{Discussion}

In previous studies on the use of actigraph, CABG patients' TST and sleep efficiency over the first 3 days after surgery were also decreased, and daytime sleep hours and awakenings were increased compared with that before surgery $[13,21]$. Consistent with these studies, we observed that patients admitted to the ICU experienced decreased nocturnal sleep time and TST, and increased day sleep time and number of awakenings. The PSQI score, which is often use as subjective measure of sleep quality, indicated poor sleep status from early postoperative week to the fourth or eighth week after discharge [12,13,21]. Similarly, the OSA-MA scores in our results showed markedly decreased sleep time and fragmented sleep on POD3 and 5. Because the preoperative

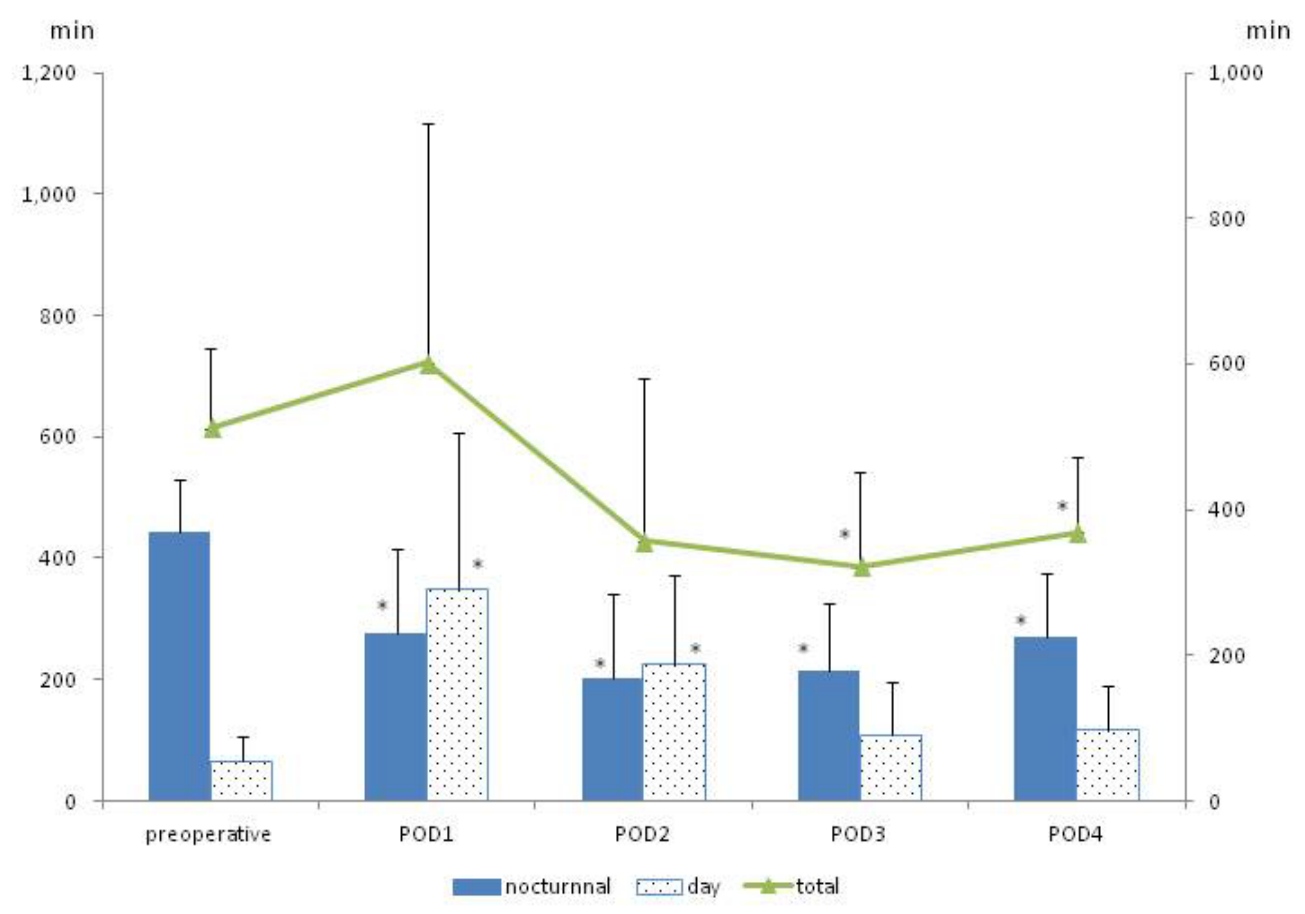

Figure 3: Distribution of nocturnal and day sleep time.

The left $y$-axes indicate nocturnal and day sleep time, the right $y$-axes indicate total sleep time. Data are expressed as mean $\pm \mathrm{SD}$. ${ }^{*} \mathrm{p}<.05$, significant difference of comparison between preoperative and each postoperative day (Bonferroni test). 


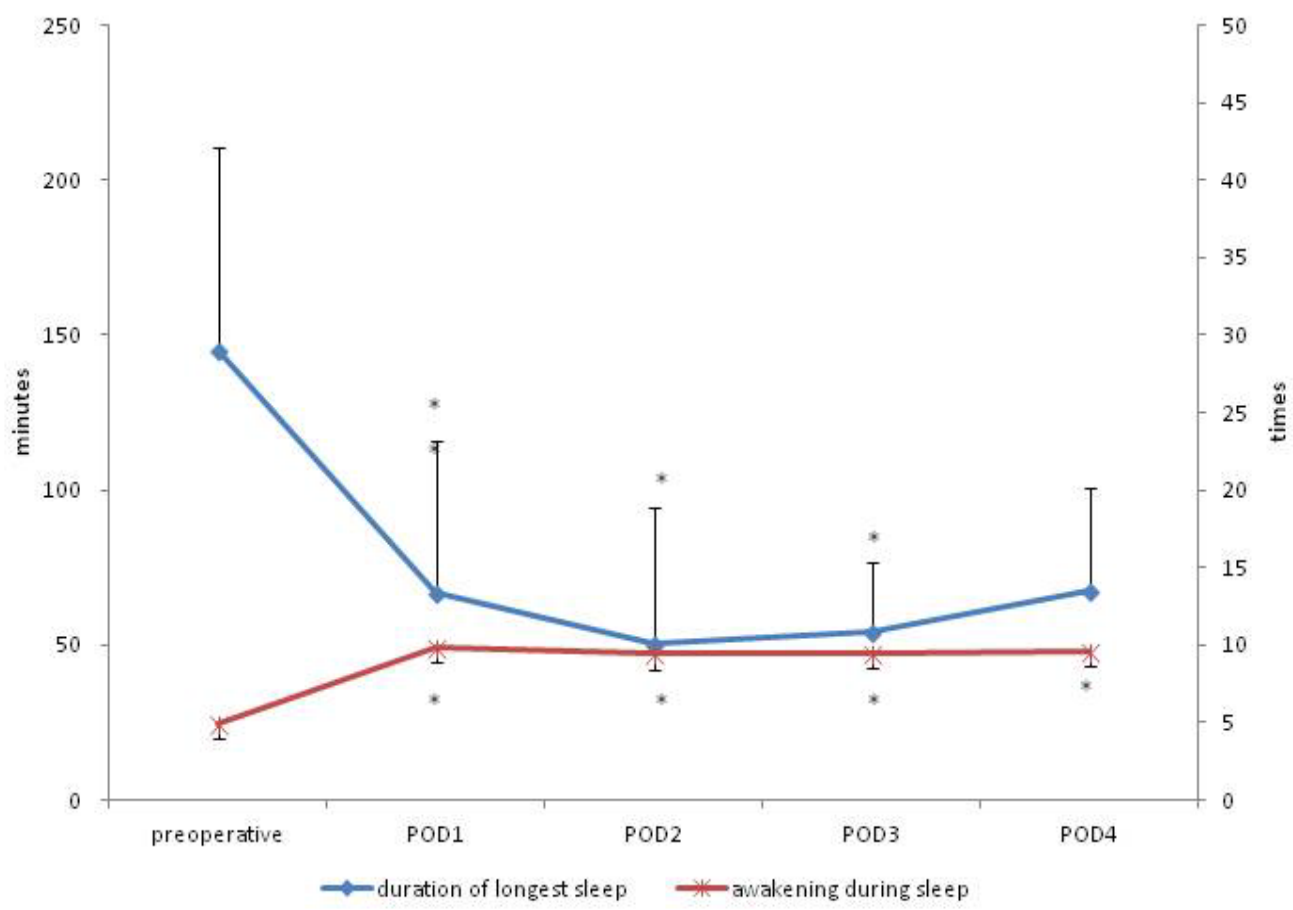

Figure 4: Duration of longest sleep and nocturnal awakenings.

The left $y$-axes indicate the duration of longest sleep times and, the right $y$-axes indicate nocturnal awakenings. Data are expressed as mean \pm SD. ${ }^{*} p<.05$ significant difference of comparison between preoperative and each postoperative day (Bonferroni test).

\begin{tabular}{|c|c|c|c|c|}
\hline & $\begin{array}{c}\text { Baseline } \\
\text { (preoperative) }\end{array}$ & POD3 & POD5 & healthy $(60-75 y e a r)^{\mathrm{a}}$ \\
\hline I, sleepiness on rising & $25.7 \pm 6.2$ & $22.3 \pm 5.6$ & $25.4 \pm 5.2$ & n.s \\
\hline $\begin{array}{c}\text { II, initiation and } \\
\text { maintenance }\end{array}$ & $18.8 \pm 5.5$ & $12.6 \pm 7.0^{*}$ & $18.8 \pm 8.5$ & Pre- $>$ POD3 (p<.05) \\
\hline III, frequent dreaming & $25.6 \pm 5.3$ & $25.8 \pm 6.5$ & $28.0 \pm 4.0$ & n.s \\
\hline IV, feeling refreshed & $22.0 \pm 7.5$ & $19.1 \pm 5.7$ & $22.1 \pm 5.4$ & $22.5 \pm 7.6$ \\
\hline V, sleep length & $25.6 \pm 5.2$ & $11.0 \pm 7.9^{*}$ & $18.7 \pm 5.1^{*}$ & $\begin{array}{c}\text { Pre- }>\text { POD3 (p<.05), } \\
\text { Pre- }>\text { POD5 (p<.05) }\end{array}$ \\
\hline
\end{tabular}

Data are expressed as mean \pm standard divisions. Lower scores indicating poor sleep quality

${ }^{*} p<.05$, significant difference of comparison between preoperative and each postoperative day

n.s, not significant

aHealthy (60-75 years) data was used from Yamamoto et al. [19]

Table 2: OSA-MA score in preoperative and postoperative day.

scores of participants were similar to those of healthy elderly [19], lower OSA-MA scores after surgery represent deterioration in the quality of sleep. This result is consistent with that estimated with the actigraph. Comparison between preoperative and postoperative data using both objective and subjective indicators revealed that reduction in and the fragmentation of nocturnal sleep did not reach preoperative levels until at least POD4. All participants in this study were transferred to private rooms in the surgical ward before the night of POD4. According to actigraph records, nocturnal sleep particularly remained poor, i.e., approximately $50 \%$ participants could not sleep at all for POD2-3. The studies measured sleep only during the postoperative period also showed that nocturnal sleep deteriorated from POD2 to POD3 [12]. Most participants had been discharged from the ICU on the period that sleep was more aggravate. After passage of 3 days from surgery, patients' physical status and environmental conditions were remedied, for example, thoracostomy tube was extubated, the frequency of nocturnal nursing care was reduced, and cardiac rehabilitation was initiated. Thus, disturbed sleep may be masked by improvement of patient's general status, whereas it continued even after transferred to the surgical ward.

ICU nurses often overestimated patients' sleep, assessing it as good sleep rather than taking the patients' perception of their sleep [16] or recording sleep patterns using polysomnography [7]. It has also been reported that nurses in the acute care setting more frequently underestimated the severity of sleep problems $[22,23]$. In addition, nursing records often only contain remarks on the patient's physical condition, and information about sleep disturbance is minimal particularly on or after the third night after surgery [17]. Our finding of poor nocturnal sleep suggests that even when patients appear to be sleeping, their sleep may be insufficient. Nurses in surgical wards must be alert for signs of life-threatening complications and for deprivation of sleep even after discharge from the ICU. However, because nurses have limited information patients' poor sleep pattern, close collaboration between the surgical ward and ICU nurses is key in providing more appropriate and early interventions. 


\section{Limitations and Further Research}

This study has the following limitations. Actigraph can be influenced by restricted body movements. The potential movement of the body is less due to postoperative pain. Nevertheless, wakefulness was estimated more than sleep in this study. Actigraph was used to determine the sleep levels in patients admitted to the ICU after surgery, and therefore, future research will require more detailed investigation of the sleep status of such patients. Another limitation, we did not determine the effect of medications on sleep, although the types and administration patterns of medication were similar among our participants. Further research is warranted to determine the effects of medication and pain on postoperative recovery of sleep patterns using a much larger sample size. In addition, it is the length of the measure period. Further investigation is required to elucidate the recovery of disrupted sleep for longer periods. Finally, light conditions were approximately controlled, although noise conditions were difficult to control in the ICU for immediate hospitalization.

\section{Conclusions}

This study demonstrated the sleep disturbance, increased number of nocturnal awakenings and decreased TST, nocturnal sleep time, duration of longest sleep, and deterioration in the quality of sleep in elderly patients after cardiac surgery. The sleep disturbances did not reach preoperative levels and persisted not only throughout patients' ICU stay but also after they had been discharged from the ICU. These findings may be provide the nurses with information for assessing of their patients' sleep, thereby develop planning and implementation of high-quality care.

\section{Acknowledgements}

This research study was supported by a Japan Society for the Promotion of Science. Grant-in-Aid for Scientific Research (C) (No. 21592709).

\section{References}

1. Krachman SL, D'Alonzo GE, Criner GJ (1995) Sleep in the intensive care unit Chest 107: 1713-1720.

2. Wood AM (1993) A review of literature relating to sleep in hospital with emphasis on the sleep of the ICU patient. Intensive Crit Care Nurs 9: 129-136.

3. Simpson T, Lee ER, Cameron C (1996) Patients' perceptions of environmental factors that disturb sleep after cardiac surgery. Am J Crit Care 5: 173-181.

4. Lane T, East LA (2008) Sleep disruption experienced by surgical patients in an acute hospital. Br J Nurs 17: 766-771.

5. Figueroa-Ramos MI, Arroyo-Novoa CM, Lee KA, Padilla G, Puntillo KA (2009) Sleep and delirium in ICU patients: a review of mechanisms and manifestations. Intensive Care Med 35: 781-795.

6. Fontana CJ, Pittiglio LI (2010) Sleep deprivation among critical care patients. Crit Care Nurs Q 33: 75-81.

7. Aurell J, Elmqvist D (1985) Sleep in the surgical intensive care unit: continuous polygraphic recording of sleep in nine patients receiving postoperative care. $\mathrm{Br}$ Med J (Clin Res Ed) 290: 1029-1032.

8. Freedman NS, Gazendam J, Levan L, Pack AI, Schwab RJ (2001) Abnormal sleep/wake cycles and the effect of environmental noise on sleep disruption in the intensive care unit. Am J Respir Crit Care Med 163: 451-457.

9. Gabor JY, Cooper AB, Crombach SA, Lee B, Kadikar N, et al. (2003)
Contribution of the intensive care unit environment to sleep disruption in mechanically ventilated patients and healthy subjects. Am J Respir Crit Care Med 167: 708-715

10. Redeker NS, Mason DJ, Wykpisz E, Glica B (1996) Sleep patterns in women after coronary artery bypass surgery. Appl Nurs Res 9: 115-122.

11. Edéll-Gustafsson UM, Hetta JE, Arén CB (1999) Sleep and quality of life assessment in patients undergoing coronary artery bypass grafting. J Adv Nurs 29: $1213-1220$.

12. Hedges C (2005) Sleep, memory, and learning in off-pump coronary artery bypass patients. Res Nurs Health 28: 462-473.

13. Yilmaz H, Iskesen I (2007) Follow-up with objective and subjective tests of the sleep characteristics of patients after cardiac surgery. Circ J 71: 1506-1510.

14. Gögenur I, Wildschiøtz G, Rosenberg J (2008) Circadian distribution of sleep phases after major abdominal surgery. Br J Anaesth 100: 45-49.

15. Hedges C, Redeker NS (2008) Comparison of sleep and mood in patients after onpump and off-pump coronary artery bypass surgery. Am J Crit Care 17: 133-140.

16. Nicolás A, Aizpitarte E, Iruarrizaga A, Vázquez M, Margall A, et al (2008) Perception of night-time sleep by surgical patients in an intensive care unit Nurs Crit Care 13: 25-33.

17. Edéll-Gustafsson U, Arén C, Hamrin E, Hetta J (1994) Nurses' notes on sleep patterns in patients undergoing coronary artery bypass surgery: a retrospective evaluation of patient records. J Adv Nurs 20: 331-336.

18. Cole RJ, Kripke DF, Gruen W, Mullaney DJ, Gillin JC (1992) Automatic sleep/ wake identification from wrist activity. Sleep 15: 461-469.

19. Yamamoto $Y$, Tanaka H, Takase M, Shirakawa S (1999) Standardization of revised version of OSA sleep inventory for middle age and aged. Brain Sci Ment Disord 10: 401-409.

20. Sachie Tabuchi, Kikuyo Koitabashi (2006) Study of the sleep patterns of patients before and after surgery-Evaluation by an actigram and an OSA sleep survey sheet (MA edition). Japanese Journal of Nursing art and Science 5: 58-68.

21. Redeker NS, Ruggiero J, Hedges C (2004) Patterns and predictors of sleep pattern disturbance after cardiac surgery. Res Nurs Health 27: 217-224.

22. Florin J, Ehrenberg A, Ehnfors M (2005) Patients' and nurses' perceptions of nursing problems in an acute care setting. J Adv Nurs 51: 140-149.

23. Lei Z, Qiongjing Y, Quli W, Sabrina K, Xiaojing L, et al. (2009) Sleep quality and sleep disturbing factors of inpatients in a Chinese general hospital. J Clin Nurs 18: 2521-2529. 